\author{
Anil K Jha ${ }^{1 \star}$ and Subekcha Karki ${ }^{2}$ \\ ${ }^{1}$ Founder Chairman Di Skin Hospital and Research \\ Centre Maharajgunj, Kathmandu, Nepal \\ ${ }^{2}$ Assistant Dermatologist to the Chair, Di Skin \\ Hospital, Maharajgunj, Kathmandu, Nepal \\ Dates: Received: 15 September, 2015; Accepted: \\ 15 October, 2015; Published: 17 October, 2015 \\ *Corresponding author: Anil K Jha, Founder \\ Chairman Di Skin Hospital and Research Centre, \\ Maharajgunj, Kathmandu, Nepal, Professor \& Head of \\ Department Nepal Medical College, Attrarkhel, Jorpati \\ Kathmandu, Nepal, E-mail: dranilkjha@hotmail.com \\ www.peertechz.com \\ ISSN: 2455-8605
}

\author{
Review Article
}

\section{Pigmentary Disorders; Vitiligo and Melasma in Context of South Asian Countries: A Psychosocio-Cosmetic Challenge}

\section{Introduction}

Vitiligo and melasma are acquired pigmentary disorder of the skin and also the leading cause for dermatologist consultation in South Asian countries like Nepal. Clinically vitiligo is characterised by well circumscribed, depigmented macules and patches secondary to selective destruction of melanocytes in the skin and mucous membrane whereas melasma is characterized by symmetrical hyperpigmented macules on the face.

\section{Vitiligo}

\section{Epidemiology}

Vitiligo vulgaris is a common clinical-type skin and mucosal disorder observed in Nepal. It can affect all skin types and affects both sexes equally however, female usually acquire the disease earlier than males. It can occur at any age, cases have been reported as early as 6 weeks after birth. Half of the patients develop the disease before the age of 20 years. Onset at an advanced age occurs although unusual and should raise concern about associated diseases like thyroid dysfunction, rheumatoid arthritis, diabetes mellitus, alopecia areata, atopic dermatitis [1-3].

With a global incidence between 1 to $2 \%$, highest incidence has been reported in India and Mexico. It accounted for $2.82 \%$ of the total outpatients seen in two referral hospitals of Nepal. Thus South-Asian countries could be a significant contributor to the global incidence of this disorder.

\section{Etiopathogenesis}

Of several pathophysiologic theories the most prominent are autoimmune phenomenon associated with underlying genetic predisposition, neurohormonal and autocytotoxic theory $[4,5]$.

\section{Presenting features}

Vitiligo presents as well circumscribed, depigmented macules and patches with convex borders, surrounded by normal skin which is slowly progressive, either by centrifugal expansion of current lesion and the appearance of a new lesion. May remain asymptomatic, may itch and have a propensity to sunburn. Koebner phenomenon develops in areas of friction \&/ or trauma. It typically appears in exposed areas such as face and hands. Chronic persistent, spontaneous repigmentation is uncommon and occurs in a perifollicular pattern. In some cases begins insidiously in sun-exposed areas during the spring and summer months. Severe sunburn, pregnancy, skin trauma, and emotional stress may precede the onset.

According to the area of involvement various types and subtypes of vitiligo are NSV (Non segmental vitiligo); focal, mucosal, acrofacial, generalised, universal, SV (segmental vitiligo); focal, mucosal, unisegmental, bisegmental or plurisegmenntal, mixed and unclassified like; focal at onset, multifocal, asymmetrical, nonsegmental, mucosal type (one site) [5].

\section{Diagnosis}

Mostly the diagnosis is made clinically. However woods lamp, a handled (UV) irradiation device or even skin punch biopsy for histopathological confirmation can be done.

\section{Review of current clinical studies of vitiligo treatments}

Although it is relatively resistant to most of the treatments, spontaneous repigmentation occurs in more than $1-25 \%$ of cases. Some studies stated occurrence of repigmentation in $10-20 \%$ of the cases.

Results of recent (since year 2009) clinical studies in vitiligo field are overviewed, with emphasis on their contribution to improved vitiligo management. The review includes all kinds of clinical studies, ranging from double-blind randomized controlled trials with objective assessment of treatment efficiency and evaluation of patient's satisfaction, to pilot trials aiming to preliminary address a question of treatment utility 
Sun protection: Sunscreen helps prevent sunburn and lessen photo damage as well as the chance that Koebner phenomenon will occur. Sunscreens also decrease tanning of the uninvolved skin and therefore lessen the contrast with vitiliginous lesions.

Cosmeceuticals: such as camouflage products and self-tanning dyes consist of three steps; including application of base cream, cover with a foundation selected for each patient then dry up with finishing powder. The newer self-tanning creams containing dihydroxyacetone are useful for light-skinned and olive complexioned patients with acral lesions [6].

Topical corticosteroids: Topical corticosteroids are useful for localized vitiligo. Class 1, 2 or 3 topical steroids showed $<20 \%$ of BSA achieving 75\% repigmentation (6-8 weeks cycle, alternate with tacrolimus) The effectiveness of intra-venous methylprednisolone (8mg/kg bodyweight) administered on 3consecutive days in patients with generalised vitiligo, showed that high-dose glucocorticosteroid pulse therapy may represent a therapeutic option in patients with generalised progressive vitiligo, and should be further evaluated in a prospective, randomised, clinical trial. In their study, $85 \%$ of the patients presenting with progressive disease showed cessation of disease progression after the infusion therapy. Repigmentation was observed in $71 \%$ of the patients with progressive vitiligo.

Calcineurin inhibitor: Lepeet al. report concluded that tacrolimus is almost as effective as clobetasol and is better for sensitive areas (e.g. eyelids) because, unlike clobetasol, it does not cause skin atrophy. The mean percentages of repigmentation were Clobetasol $49.3 \%$ and Tacrolimus $41.3 \%$. In their study, $90 \%$ of patients experienced some repigmentation after 2 months treatment. Tacrolimus was observed to be the most effective on head and neck lesions compared to trunk and extremities, with shorter disease duration and darker skin photo type predicting better response. In addition, response to tacrolimus monotherapy appeared to be better in children. In addition van Geel et al. studied experimentally induced koebnerization and also stressed a necessity of early treatment during inflammatory phase accompanying koebnerization. In this study, in line with others observation, both tacrolimus and mometasone furoate were equally efficient in halting spread of the lesions. Tacrolimus in combination with oral prednisolone was efficient in treatment of newly manifested vitiligo. Pimecrolimus was less efficient than topical mometasone in paediatric vitiligo treatment (mean repigmentation $42 \%$ vs. $65 \%$ ), with pimecrolimus being efficient only on head and neck, whereas mometasone could be used for treatment of any part of the body [7].

We usually prescribe Tacrolimus at $0.03 \%$ preparation alone or in combination with mid-potency steroid resulting in repigmentation among $60-80 \%$ of cases and also help stabilize the progression of the lesions.

Vitamin-D derivative: A number of studies have recently reported that the treatment with vitamin D compounds or their combination with ultraviolet light or corticosteroid enhances repigmentation in vitiligo; however, the casual relationship at the cellular and molecular levels has not so far been investigated. Calcipotriol plus photochemotherapy also appears very promising but certainly requires further evaluation. In a pilot study, topical calcipotriene and betamethasone dipropionate is a promising combination to treat facial vitiligo, but because of lack of control group, the definite conclusion is hard to be made [8].

Phototherapy: (UV-A, narrowband UV-B), Photochemotherapy (psoralen plus UV-A [PUVA]), Psoralen with sunlight (PUV Asol)

Phototherapy is an acknowledged first-line option in generalised vitiligo treatment. Topical and systemic PUVA therapy may require 100-300 treatment sessions to achieve complete repigmentation .NBUVB is as efficient as topical PUVA but with fewer adverse effects (given 2-3 X/week with starting dose of 100-250 $\mathrm{mJ} / \mathrm{cm}^{2}$, increased in increments of $10-20 \%$ until mild asymptomatic erythema achieved). The major side effects of photochemotherapy are severe phototoxic reaction, blistering, hyperpigmentation, photo-allergic reaction, hyperkeratosis of lesional skin, skin malignancies.

NBUVB can be used in children, pregnant or lactating women and patients with hepatic or kidney dysfunction. The combination of PUVA and calcipotriol is highly effective and works faster and may be used for shortening the therapy with PUVA in the treatment of vitiligo. Oral PUVA using 8 -MOP $(0.4-0.6 \mathrm{mg} / \mathrm{kg})$ administered $2 \mathrm{X} /$ week; initial dose of UVA is usually $0.5-1.0 \mathrm{~J} / \mathrm{cm}^{2}$. Visible light (635nm low energy laser) for SV treatment resulted in 7out of 14 patients responding to the treatment (response was defined as achieving at least $25 \%$ of repigmentation) thus confirming efficiency of visible light in SV treatment.

In our context also NBUVB seems to provide excellent results among patients with generalized, progressive type of vitiligo; one of the limitations is the frequent hospital visits.

In stable, localised vitiligo topical 8-methoxy psoralen along with appropriate sun exposure are showing good results.

\section{Surgical techniques}

Cosmetic tattooing is used for localised stable vitiligo, especially of the mucosal type. Pigments used in cosmetic tattoo are inert iron oxides that are available in more than 15shades. The colour is implanted into the dermal layer with specialised techniques and cannot be washed off. Of the general tendency, younger patients respond usually better, with facial and trunk lesions repigmenting better than those with acral location. Dermatosurgical procedures offer a good alternative and option of management in depigmentary disorders. However, it is important to counsel the patients regarding the disease, procedures and their expected outcome, and follow strict criteria while selecting patients, so that the outcome is satisfactory both for the doctor and the patient. The choice of procedure must be individualized, considering the size of the lesion to be treated, and readily available facilities [3,5,9-11].

Procedures like punch grafting and split thickness skin grafting or epidermal grafting are inexpensive, relatively simple to perform and thus can be performed as office procedures. These techniques can be modified to minimize known adverse effects and achieve superior results. Side effects noted were like occasional perigraft halo, hypertrophic scar, koebnerization, hypo or hyperpigmentation 2 year follow up of 49 patients who underwent autologous non-cultured epidermal method to treat pediatric vitiligo. 
At our hospital we have experienced excellent outcome among our patients especially with stable localized vitiligo areas not responding to topical treatments.

Dermatosurgical procedures produce better results when combined with post-operative PUVA and should be advised for all patients.

There is scope for improving the technique of split-thickness skin grafting to harvest uniformly thin grafts using simple hand held dermatome. This technique would ensure an inexpensive, time saving procedure with equally well, if not excellent, cosmetic outcome. Autologous cultured epidermal grafting (CEA) containing only melanocytes or both keratinocytes and melanocytes used either as sheets or cell suspensions have proved to be effective in treating stable vitiligo but are laboratory dependent. This has eliminated the need of TCL and has been adapted to successfully repigment stable vitiligo lesions. With the introduction of autologous cell harvesting devices (Recell Kit) the methodology is further simplified $[3,5,6]$

Excimer laser: The most recent effective and approved therapy for vitiligo is the 308-nm excimer laser with or without topical calcineurin antagonists. Efficiency was revealed for excimer laser treatment in; children with $50 \%$ of treated lesions achieved 50\%repigmentation, adults $60.4 \%$ of patches repigmented for more than $75 \%$. Besides surgical methods EL might be an option for SV patients. For vitiligo universalis, treatment with topical 4-methoxyphenol and Q-switched (QS) ruby laser has been effective. It destroys the melanosome in melanocytes and keratinocytes by selective photothermolysis [12-14].

Combination therapies: In controlled trial tacrolimus vs. fluticasone propionate, mometasone furoate and clobetasol propionate in children with general conclusion of similar efficiency of steroid and tacrolimus. Both agents showed poor results when SV lesion was treated. Combination of topical tacrolimus, targeted NBUVB and systemic prednisolone showed promising results in recently onset UV resistant areas, such as bony prominences of the extremities.

In combination with NBUVB, tacrolimus showed better results compared to placebo. There were potential benefits gained from combination of Monochromatic excimer laser (MEL) and topical tacrolimus to enhance clinical response.

Double blind studies show that pimecrolimus $1 \%$ cream combined with NBUVB is superior to placebo, especially for facial lesions. Combining blister roof transplantation (BR) with khellin in liposomes and ultraviolet light (KLUV) in the treatment of recalcitrant vitiligo patches [12-14].

Complementary therapies: Ginkgo Biloba and leflunomide have immune modulating properties. In a pilot study of Szczurko et al., potency of oral Ginkgo biloba extract to halt the progression of active vitiligo has been evaluated. Previously, statistically significant cessation of the disease activity was observed in placebo controlled clinical trial. Disease progression was stopped in a 12 -week follow up after leflunomide therapy. At our OPD Ginkgo Biloba are also prescribed and have proven to help stabilize the progression of disease to quite an extent. It is a very good alternative to usually prescribed oral steroids.
Pseudocatalase creams with Dead Sea climatotherapy are also compatible with repigmentation. Analysis of a large cohort study of patients revealed that $85 \%$ patients responded to the therapy. The authors concluded that Dead Sea climatography is an effective treatment modality of vitiligo with relatively few side effects, Topical melagenina 1 and 2 solution manufactured in Cuba at Cetro de Histoterapia, Havana is an alcoholic extract from human placenta, containing placental lipoprotein fraction. Its active ingredients are melagenina and calcium chloride. It generally works by triggering the production of melanocytes [12-14].

Oral L-phenylalanine is a product of Venezuela, VitilVenz, containing coenzyme Q10, vitamins C and E and other natural stimulators of mitochondria was tested in 100 patients and control subjects with positive results.

Patients with extensive disease ( $>50 \%$ body area) who desire permanent matching of the skin colour but for whom repigmentation is not possible can be depigmented with $20 \%$ monobenzyl ether of hydroquinone, 2times daily for 9-12months.

\section{Natural treatments}

Vitiligo oil compounds such as psoralea Coryli Folia/ breadroot, coconut oil, kala jeera/ nigella/ black cumin, berberis vulgaris/ barberry root/ trailing mahonia, St. John Wort are commonly used herb for this condition; the herb oil can be applied to the skin [12-14].

Wild Duckweed (Lemna Minor) works by reactivating melanocytes.

Bergamot essential oil has substances in it called Bergaptenes that have strong photosensitising action may help turn back on melanocytes which are activated by ultraviolet light, pepper has direct antioxidant activity against various free radicals and scientists recently discovered that can even stimulate colouration of the skin in an animal model vitiligo.

\section{Melasma}

\section{Epidemiology}

Melasma predominantly affects Fitzpatrick skin prototype 3-4 and often lasts for many years after pregnancy. In Southeast Asia, the prevalence has been reported to be as high as $40 \%$ in females and $20 \%$ in males. In a study by B Dwari, S Palaian, A Poudel, S Prabhu clinical profile and management pattern of melasma patients in Western Nepal: A Hospital Based Study concluded the clinical profile and treatment pattern of the melasma patients were analyzed. Majority of the patients were females and were of child bearing age. Sunscreens were the most commonly used medications and the drug therapy was associated with huge economic impact.

The prevalence of melasma is unknown. Studies from Mexico and Peru found that melasma accounted for 4 to $10 \%$ of new dermatology hospital referrals. It is more common in women than in men and is rare before puberty. Melasma occurs during pregnancy in approximately $25 \%$ of cases. Among pregnant women, the prevalence of melasma ranges between 15 and $50 \%$.

Although no race is spared, melasma is far more common in 
women and in dark skinned individuals. It is a common and growing cosmetic concern among Nepalese population with Fitzpatrick skin types III-V.

In a retrospective study conducted by Anil K. Jha [15], Amatya Benl, Amatya Amit, Pattern of Skin Diseases in Kathmandu Nepal, Nepal Journal of Dermatology, Venereology and Leprology; melasma composed the third most common dermatological affection. The total population included 3224, new dermatological cases were $235,7.29 \%$ cases of melasma were seen over a period of one year; 203 (12.69\%) were females and 32 (1.93\%) were males [16].

\section{Clinical and pathologic features}

Melasma presents as symmetrical hyperpigmentation appearing as light brown to dark, muddy brown macules and patches on the face, especially the forehead, cheeks, nose, upper lip or chin. The authors found 2basic forms; Epidermal form lesions which enhance when viewed under wood lamp, featuring melanin deposition mainly in the basal and suprabasal layers and dermal form with superficial and deep perivascular melanophages in the dermis which do not enhance implying an increase in dermal melanin content.

Dermoscopy can be used for the diagnosis of melasma. Lesions of melasma show diffuse reticular pigmentation in various shades of brown with sparing of follicular openings. Dermal melasma shows diffuse dark brown to greyish pseudo reticular pigmentation. Annular, honeycomb and arcuate structures may be seen.

\section{Etiopathogenesis}

Studies have confirmed that both melanocytosis (increased number of melanocytes) as well as increased melanogenesis (increased production of melanin) are responsible for the hyperpigmentation in melasma. Kim et al. carried out a histological study to evaluate the vascular characteristics of melisma [17]. They found that vascular endothelial growth factor was increased in lesional skin in melasma signifying increased vascularization. This has clinical implications and indicates the need to devise treatment options to target the vascular component in melasma. In a prospective comparative splitface randomized study carried out by Passeron et al., it was found that combination of TCC and pulsed dye laser (PDL) was more effective than TCC alone [18]. The combination treatment also prevented the relapses.

Kang et al. performed a transcriptional analysis in melasma skin samples and found that 279 genes were stimulated and 152 were found to be down regulated. Many melanin biosynthesis related genes as well as melanocyte markers such as TYR, MITF, SILV, and TYRP1 were found to be up regulated in melasma skin [19].

Several other genes involved in other biological pathways were found to be affected. These include Wnt pathway modulation genes, genes involved in prostaglandin synthesis and fatty acid metabolism [20].

Another interesting finding was the role of $\mathrm{H} 19$ gene in melasma pathogenesis as studied by Kim et al. [20], H19 gene transcribes a noncoding ribonucleic acid (RNA) and is found to be down regulated in melasma lesions. This induces stimulation of melanogenesis and increased transfer of melanin from melanocytes to keratinocytes.

iNOS and nuclear factor-kappa B pathways have also been found to be implicated in melasma pathogenesis. In a study by Jo et al. iNOS expression was found to be increased in melasma lesions [21].

The most affected biological process in melasma is lipid metabolism [20]. Lipid metabolism genes, such as peroxisome proliferator-activated receptor alpha (PPAR), arachidonate 15lipoxygenase, PPAR gamma coactivator 1 alpha, type B (ALXO 15B), diacylglycerol o-acyltransferase 2-like 3 were found to be down regulated. This down regulation is caused by chronic UV exposure [22]. Another change seen in melasma skin is thinning of the stratum corneum (SC) as found in skin biopsies that show rete ridge flattening and epidermal thinning? SC thinning coupled with disturbed lipid synthesis is responsible for the impaired SC integrity and a delayed barrier recovery rate seen in melasma skin [24].

In a study by Lee et al. [23], melanin index, erythema index, and SC hydration were found to be significantly higher in lesional melasma skin as compared to perilesional normal skin. This proves that prominent vascularization accompanies hyperpigmentation [24].

Prolonged UV exposure-induced dermal inflammation and fibroblast activation may upregulate stem cell factors in the melasma dermis, causing increased melanogenesis.

The above findings emphasize the need for devising treatment options targeting these pathogenic factors in melasma.

\section{Review of current clinical studies of melasma treatments}

Sunscreen and camouflage: Sunscreen plays a vital role for melasma prevention as well as control and affects the overall outcome of any treatment options for patients. Depending upon the skin type and sun exposure appropriate SPF (sun protection factor) are prescribed and advised to be used on regular basis. Camouflage is advised for patients who need temporary coverage of the hyperpigmentation. It is best to advice camouflage whenever required but always along with sunscreen.

Phenolic compounds: Current role of Hydroquinone (HQ): HQ is a dihydric phenol that inhibits the conversion of dopa to melanin by inhibiting the tyrosinase enzyme. Other proposed mechanisms of action include the inhibition of RNA and deoxyribonucleic acid synthesis and destruction of melanocytes.

HQ is considered the gold standard of treatment for melasma. It is used at a concentration of 2-4\%. Its efficacy is enhanced when used in combination with other agents. These combinations include the Kligman's formula ( $5 \% \mathrm{HQ}, 0.1 \%$ tretinoin, and $0.1 \%$ dexamethasone), modified Kligman's formula (4\% $\mathrm{HQ}, 0.05 \%$ tretinoin and $1 \%$ hydrocortisone acetate), Pathak's (2\% HQ and $0.05-$ $0.1 \%$ tretinoin) and Westerhof's formula $(4.7 \% \mathrm{~N}$-acetylcysteine, $2 \%$ HQ and $0.1 \%$ triamcinolone acetonide) [25]. Combination of microencapsulated HQ $4 \%$ with retinol $0.15 \%$ and antioxidants has also been tried in melasma with studies showing improvement in 
disease severity, pigmentation intensity, lesion area and colorimetry assessments [26].

In a randomized, split-face study, Monheit and Dreher compared the safety and efficacy of skin lightening cream (containing HQ 4\% and four skin brightening actives) with a TCC (containing $4 \% \mathrm{HQ}$, $0.05 \%$ tretinoin, and $0.01 \%$ fluocinolone acetonide) in 20 Caucasian female patients with melisma [26]. They found that after 4 weeks once daily treatment, the skin lightening cream SLC was comparable in both efficacy and safety to the well-established TCC treatment for melasma. One-third of patients experienced local side effects in the form of erythema, peeling of skin and dryness with both the creams.

In a randomized, controlled study Arellano et al. [27], compared the clinical efficacy and safety of two 6-month triple combination (containing HQ, fluocinolone acetonide and tretinoin) maintenance regimens in 242 Brazilian and Mexican patients with moderate-tosevere melisma [27]. Global severity score, melasma area severity index (MASI), and quality of life questionnaire were used to assess the efficacy of treatment. After eight weeks of daily TCC application, subjects were randomized to receive TCC in a twice weekly or tapering regimen $\left(3 /\right.$ week $\left[1^{\text {st }}\right.$ month $], 2 /$ week $\left[2^{\text {nd }}\right.$ month $], 1 /$ week [ $4^{\text {th }}$ month]) for 6 months. They found that twice-weekly regimen showed better effectiveness on postponing relapse in patients with severe melasma. About $53 \%$ of patients were relapse-free after 6 months.

Tretinoin: Tretinoin is widely used for the treatment of melasma as an over-the-counter lightening agent. Considering its efficacy, peeling with tretinoin may improve pigmentation in melasma. Faghihi et al. performed a randomized, double-blinded clinical trial to compare the efficacy of $1 \%$ tretinoin peel versus $70 \%$ GA peel in 63 female patients with bilateral melisma [28]. It was found that the efficacy of $1 \%$ tretinoin peel was similar to $70 \%$ GA. Post procedure discomfort as expressed by the patients was significantly lower with $1 \%$ tretinoin.

Tretinoin can also be used as a peeling mask. Ghersetich et al. evaluated the efficacy of $10 \%$ tretinoin peeling mask using standardized digital photos, mexameter measurement and MASI evaluation in 20 female patients with Fitzpatrick skin type II-VI [29]. They found moderate or marked improvement in all patients using these three parameters without any adverse events. At 10 weeks, the difference in the average MASI calculated at baseline and after 10 weeks were 2.9.

Combination products: (Hydroquinone + Retinoids + Topical steroids), few other commonly used topical agents are Azelaic acid, Kojic acid, Ascorbic acid, Licorice extract, Soy.

Chemical peels: commonly used peels for melasma are AHA (Alpha Hydroxy Acid), SSA (Salicylic acid), Jessner solution (SSA+Lactic Acid+ Resorcinol+Ethanol), 10\%-50\% TCA (Trichloroacetic acid) peel.

In current practice and from experience chemical peels do not give satisfactory result, especially because of the skin type of our patients. We would not advise chemical peels due to chances of post inflammatory hyper \&/or hypopigmentation that are very common in Skin type III-V.
Laser and Light therapies: Various lasers that have been used for the treatment of melasma are:

- Green light: Flashlamp-pumped PDL (510 nm), frequency doubled Q switched neodymium: Yttrium aluminium garnet-532 nm (QS Nd: YAG)

- Red light: Q switched ruby (694 nm), Q switched alexandrite $(755 \mathrm{~nm})$

\section{- Near-infrared: QS Nd: YAG (1064 nm).}

Fractional lasers have been used for the treatment of pigmented lesions, including melasma.

QS Nd: YAG is the most widely used laser for the treatment of melasma. The fluence used is less than $5 \mathrm{~J} / \mathrm{cm}^{2}$, spot size $6 \mathrm{~mm}$, and frequency of $10 \mathrm{~Hz}$. The number of treatment sessions varies from 5 to 10 at 1 -week intervals.

Nowadays, technique called "laser toning" or "laser facial" has become increasingly popular for the treatment of melasma. This involves the use of a large spot size $(6-8 \mathrm{~mm})$, low fluence (1.6-3.5 J/ $\mathrm{cm}^{2}$ ) and multiple passes.

QS 1064 nm Nd: YAG laser performed every 1-2 weeks for several weeks [30], although good efficacy has been seen with this technique, side effects have also been reported. These include hypopigmentation, depigmentation, rebound hyperpigmentation, physical urticaria, acneiform eruption, petechiae, and herpes simplex reactivation. Combination of ablative and pigment selective lasers has also been tried in melasma. Ablative lasers remove the epidermis containing excess melanin; this is followed by the use of Q switched pigment selective laser that can target the dermal melanophages. Angsuwarangsee and Polnikorn studied the efficacy of combined ultrapulse $\mathrm{CO}_{2}$ laser and $\mathrm{Q}$ switched alexandrite laser (QSAL) alone in six patients with melasma. The site that received combination treatment showed significant response compared with the site that was treated with QSAL alone [30]. However, side effects in the form of contact dermatitis and hyperpigmentation were observed in few patients, especially in those with dark skin. Hence, the use of combination of lasers should only be used for refractory melasma.

\section{On the horizon}

4-n-Butylresorcinol is a derivative of resorcinol that inhibits tyrosinase and tyrosinase related protein enzymes in the melanin biosynthetic pathway [31], Studies have shown good efficacy and safety with 4-n-butylresorcinol in patients with melisma [32]. Huh et al. [34], conducted a randomized controlled split-face trial in 23 patients with melasma to evaluate the efficacy and safety of liposome-encapsulated 4 -n-butylresorcinol $0.1 \%$ cream. They found a statistically significant reduction $(P<0.043)$ in the melanin index on the treatment side [33].

Tranexamic Acid (TA) is Trans-4-(aminomethyl) cyclohexane carboxylic acid, a lysine analog that is in use as an antifibrinolytic agent for over 30 years. It inhibits UV-induced plasmin activity in keratinocytes by preventing the binding of plasminogen to the keratinocytes. This results in less free arachidonic acid and a diminished ability to produce prostaglandins, and this decreases 
melanocyte tyrosinase activity. Zhang et al. demonstrated that TA can interfere with the catalytic reaction of tyrosinase and inhibit melanogenesis [34]. In the treatment of melasma TA can be used orally, topically or by intradermal microinjection. As a hemostatic, TA is prescribed in a dose of $1000 \mathrm{mg} 3$ times daily, whereas in the treatment of melasma, it is used in a dose of $250 \mathrm{mg}$ twice daily [35].

In a study conducted by $\mathrm{Wu}$ et al., 74 Chinese patients with melasma were treated with TA $250 \mathrm{mg}$ twice daily for 6 months. The effects of treatment were evaluated by two physicians and by the patients based on the reduction in melasma size and improvement of pigmentation. The results were excellent in $10.8 \%$, good in $54 \%$, fair in $31.1 \%$ and poor in $4.1 \%$. Side effects seen were gastrointestinal discomfort and hypomenorrhea. Recurrence was seen in 9.5\% patients [36].

TA can also be used topically. In a recent double-blind randomized prospective study by Kanechorn et al., 5\% TA incorporated in a liposome gel formulation was used in 23 Asian women with bilateral epidermal melasma. The treatment was given for 12 weeks and compared with the vehicle in a split-face trial [16,37]. 78.2\% of patients showed a decrease in the melanin index. However, results were not significant as compared with vehicle. Another finding seen in this study was the erythema induced by topical TA.

In a study conducted by Lee et al. [39], in 100 Korean women with melasma, TA was used intradermally $(4 \mathrm{mg} / \mathrm{ml})$ every week for 12 weeks [38]. The treatment caused a significant decrease in MASI score (13.22 vs. 9.02 at week 8 and vs. 7.57 at week $12, \mathrm{P}<0.05$ for both) with minimal side effects.

The advantage with TA is its good safety profile. Furthermore, it is temperature stable, UV insensitive and does not get oxidized easily. More studies are needed to evaluate its anti-melasma potential.

Similarly in our current practice the result of patients undergoing treatment with oral tranexamic acid $500 \mathrm{mg}$ once daily dosage is excellent. Improvement is expected and seen with an average of 1 to 3 months period.

\section{Conclusion}

Although not a disease in itself, vitiligo and melasma are the most common challenges dermatologists have to manage on daily basis. The treatment should not only focus on the superficial level but focus on various morbidities associated with it such as physical, social, cosmetic as well as psychological. The challenge is to make the patient as well as the society realize that; these are pigmentary disorder conditions rather than disease in itself. Patients may be reassured that the disorders are not life threatening or do not lead to physical disability, but it is well known that it can lead to drastic psychosocial consequences, especially in our part of the world .It is well understood in regions like Europe and America with skin type 1 due to lack of colour contrast vitiligo is not much given concern but melasma and other hyperpigmentary disorders remain a common dermatological challenge.

\section{References}

1. Jha AK, Pandey SS, Gulati AK, Bansal V, Shukla VK, et al. (1993) Inoculation of a Cultured Autologous Epidermal Suspension Containing Melanocytes In Vitiligo. Arch Dermatol 129: 785-786.

2. Anil K Jha, Amatya Benl, Amatya Amit. Pattern Of Skin Diseases In Kathmandu Nepal, Nepal Journal Of Dermatology, Venereology And Leprology.

3. Jha AK, Gurung D (2006) Seasonal Variation Of Skin Diseases In Nepal: Hospital Based Annual Study Of Out-Patients Visit. Nepal Med Coll J 8: 266268.

4. Jean L Bolognia (2012) Dermatology, $3^{\text {rd }}$ Edition 1:

5. (2011) Jam Acad Dermatology.

6. Birlea SA, Costin GE, Norris DA (2009) New Insights On Therapy With Vitamin D Analogs Targeting The Intracellular Pathways That Control Repigmentation In Human Vitiligo. Med Res Rev 514-546.

7. Parsad D, Saini R, Verma N (1998) Combination Of Puvasol And Topical Calcipotriol In Vitiligo. Dermatology 197: 167-170.

8. Wong R, Lin AN (2013) Efficacy of Topical Calcineurin Inhibitors in Vitiligo. Int Journal Of Dermatology 52: 491-496.

9. Jha AK, Pandey SS, Shukla VN (1994) Punch Grafting In Vitiligo. Ind J Dermatology Venerology And Leprology 60:188-192.

10. Sarveswari KN (2010) Cosmetic Camouflage In Vitiligo. Indian J Dermatol 5 : 211-214.

11. Mohanty S, Kumar A, Dhawan J, Sreenivas V, Gupta S (2011) Non Cultured Extracted Hair Follicle Outer Root Sheath Cell Suspencion For Transplantation In Vitiligo. British Journal Of Dermatology 164: 1241-1246.

12. National Institute Of Health (2010) Department Of Health \& Human Services, National Institute Of Arthritis And Musculoskeletal And Skin Disease, Last Updated 8/1/2010.

13. Igor V Korobko (2012) Review Of Current Clinical Studies Of Vitiligo Treatment. Dermatologic Therapy 25: S17-S27.

14. Taieb A, Alomar A, Böhm M, Dell'anna ML, De Pase A, et al. (2013) Guidelines For The Management Of Vitiligo: The European Dermatology Forum Consensus, Br J Dermatol 168: 5-19.

15. Jha AK, Gurung D (2013) Dermatosurgical Management Of Leukoderma; Punch Grafting Vs. Split-Thickness Skin Grafting. Br J Dermatol 168: 5-19.

16. Taieb A, Alomar A, Böhm M, Dell'anna ML, De Pase A et al. (2013) Vitiligo European Task Force (VETF); European Academy Of Dermatology And Venereology (EADV); Union Europe'Enne Des Me'Decins Spe'Cialistes (UEMS) 168: 5-19.

17. Kim EH, Kim YC, Lee ES, Kang HY (2007) The vascular characteristics of melasma. J Dermatol Sci 46: 111-116.

18. Passeron T, Fontas E, Kang HY, Bahadoran P, Lacour JP, et al. (2011) Melasma treatment with pulsed-dye laser and triple combination cream: A prospective, randomized, single-blind, split-face study. Arch Dermatol 147: 1106-1108.

19. Kang HY, Suzuki I, Lee DJ, Ha J, Reiniche P, et al. (2011) Transcriptional profiling shows altered expression of wnt pathway- and lipid metabolismrelated genes as well as melanogenesis-related genes in melasma. J Invest Dermatol 131: 1692-1700.

20. Kim NH, Lee CH, Lee AY (2010) H19 RNA downregulation stimulated melanogenesis in melasma. Pigment Cell Melanoma Res 23: 84-92.

21. Jo HY, Kim CK, Suh IB, Ryu SW, Ha KS, et al. (2009) Co-localization of inducible nitric oxide synthase and phosphorylated Akt in the lesional skins of patients with melasma. J Dermatol 36: 10-16.

22. Kang WH, Yoon KH, Lee ES, Kim J, Lee KB, et al. (2002) Melasma: Histopathological characteristics in 56 Korean patients. Br J Dermatol 146: 228-237.

23. Lee DJ, Lee J, Ha J, Park KC, Ortonne JP, et al. (2012) Defective barrier function in melasma skin. J Eur Acad Dermatol Venereol 26: 1533-1537. 
24. Cestari TF, Hexsel D, Viegas ML, Azulay L, Hassun K, et al. (2006) Validation of a melasma quality of life questionnaire for Brazilian Portuguese language: The MelasQoL-BP study and improvement of QoL of melasma patients after triple combination therapy. Br J Dermatol 156:13-20.

25. Cook-Bolden FE, Hamilton SF (2008) An open-label study of the efficacy and tolerability of microencapsulated hydroquinone $4 \%$ and retinol $0.15 \%$ with antioxidants for the treatment of hyperpigmentation. Cutis 81: 365-371.

26. Monheit GD, Dreher F (2013) Comparison of a skin-lightening cream targeting melanogenesis on multiple levels to triple combination cream for melasma. $\mathrm{J}$ Drugs Dermatol 12: 270-274.

27. Arellano I, Cestari T, Ocampo-Candiani J, Azulay-Abulafia L, Bezerra Trindade Neto P, et al. (2012) Preventing melasma recurrence: Prescribing a maintenance regimen with an effective triple combination cream based on long-standing clinical severity. J Eur Acad Dermatol Venereol 26: 611-618.

28. Ghersetich I, Troiano M, Brazzini B, Arunachalam M, Lotti T (2010) Melasma: Treatment with 10\% tretinoin peeling mask. J Cosmet Dermatol 9: 117-121.

29. Hong SP, Han SS, Choi SJ, Kim MS, Won CH, et al. (2012) Split-face comparative study of $1550 \mathrm{~nm}$ fractional photothermolysis and trichloroacetic acid $15 \%$ chemical peeling for facial melasma in Asian skin. J Cosmet Lase Ther 14: 81-86.

30. Chan NP, Ho SG, Shek SY, Yeung CK, Chan HH (2010) A case series of facial depigmentation associated with low fluence Q-switched 1,064 nm Nd: YAG laser for skin rejuvenation and melasma. Lasers Surg Med 42: 712-719.

31. Angsuwarangsee S, Polnikorn N (2003) Combined ultrapulse CO 2 laser and Q-switched alexandrite laser compared with Q-switched alexandrite lase alone for refractory melasma: Split-face design. Dermatol Surg 29: 59-64.

32. Kim DS, Kim SY, Park SH, Choi YG, Kwon SB, et al. (2005) Inhibitory effects of 4-n-butylresorcinol on tyrosinase activity and melanin synthesis. Biol Pharm Bull 28: 2216-2219.

33. Khemis A, Kaiafa A, Queille-Roussel C, Duteil L, Ortonne JP (2007) Evaluation of efficacy and safety of rucinol serum in patients with melasma: A randomized controlled trial. Br J Dermatol 156: 997-1004.
34. Huh SY, Shin JW, Na JI, Huh CH, Youn SW, et al. (2010) Efficacy and safety of liposome-encapsulated 4 -n-butylresorcinol $0.1 \%$ cream for the treatment of melasma: A randomized controlled split-face trial. J Dermatol 37: 311-315.

35. Zhang $X$, Yang X, Yang H, Yang Y (2003) Study of inhibitory effect of acidum tranexamicum on melanin synthesis. Chin J Dermatovenerol Integr Tradit West Med 2: 227-229.

36. Dunn CJ, Goa KL (1999) Tranexamic acid: A review of its use in surgery and other indications. Drugs 57: 1005-1032.

37. Wu S, Shi H, Wu H, Yan S, Guo J, et al. (2012) Treatment of melasma with oral administration of tranexamic acid. Aesthetic Plast Surg 36: 964-970.

38. Kanechorn Na Ayuthaya P, Niumphradit N, Manosroi A, Nakakes A (2012) Topical 5\% tranexamic acid for the treatment of melasma in Asians: A double blind randomized controlled clinical trial. J Cosmet Laser Ther 14: 150-154.

39. Lee JH, Park JG, Lim SH, Kim JY, Ahn KY, et al. (2006) Localized intradermal microinjection of tranexamic acid for treatment of melasma in Asian patients: A preliminary clinical trial. Dermatol Surg 32: 626-631.

40. Reza Yahgoobi, Mohammad Omidian Bagherani (2011) Vitiligo: A Review Of The Published Work, Journal Of Dermatology 38: 419-431.

41. K Ezzedine, HW Lim, T Suzuki, I Katayama, I Hamzavi, et al. (2012) And On Behalf Of The Vitiligo Global Issue Consensus Conference Panellists, Pigment Cell \& Melanoma Research 25: E1-E13.

42. De Leeuw J, Assen YJ, Van Der Beek N, Bjerring P, Martino Neumann HA (2011) Treatment In Vitiligo With Khellin Liposomes, Ultraviolet And Blister Roof Transplantation. J Eur Acad Dermatol Venereol 25: 74-81.

43. Alikhan A, Felsten LM, Daly M, Petronic-Rosic V (2011) Vitiligo: a comprehensive overview Part I. Introduction, epidemiology, quality of life, diagnosis, differential diagnosis, associations, histopathology, etiology, and work-up, Part 1. J Am Acad Dermatol 65: 473-491.

44. Rashmi Sarkar, Pooja Arora, Vijay Kumar Garg, Sidharth Sonthalia, Narendra Gokhale (2014) Melasma update. Indian Dermatol Online J 5: 426-435.

Copyright: (c) 2015 Jha AK, et al. This is an open-access article distributed under the terms of the Creative Commons Attribution License, which permits unrestricted use, distribution, and reproduction in any medium, provided the original author and source are credited. 